\title{
CORRECTION
}

\section{Correction to: Phase field fracture in elasto-plastic solids: a length-scale insensitive model for quasi-brittle materials}

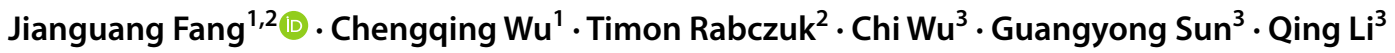

Published online: 22 April 2021

๑) Springer-Verlag GmbH Germany, part of Springer Nature 2021

\section{Correction to: Computational Mechanics (2020) 66:931-961 https://doi.org/10.1007/s00466-020-01887-1}

The original article contained typographical errors that a number of double brackets $\llbracket \rrbracket$ were missing in Sect. 3.3 and Table 2.

In Sect. 3.3, $d^{\prime}(0), \Lambda(0)$ and $u(0)$ should be replaced by $\llbracket\left[d^{\prime}\right](0), \llbracket \Lambda \rrbracket(0)$ and $\llbracket u \rrbracket(0)$, respectively, in Eqs. (40)-(42) and related paragraphs on Pages 938 and 940 . The corrected equations read:

$\left.\llbracket\left[d^{\prime}\right]\right](0)=-\frac{2}{l_{c}} \sqrt{H(\sigma, d(0), C)}$

$\llbracket \Lambda \rrbracket(0)=\frac{\left.-g_{f} l_{c}\left[\llbracket d^{\prime}\right]\right](0)}{2[1-d(0)] \sigma_{y 0}}=\frac{[1-d(0)] g_{f} \sqrt{H(\sigma, d(0), C)}}{\sigma_{t}}$

$$
U=2 \int_{0}^{L / 2} \frac{\sigma}{(1-d)^{2} E_{0}} d x+\llbracket \Lambda \rrbracket(0)
$$

In Table 2, the governing equations in the singular domain $\Gamma$ need to be corrected as:

\begin{tabular}{lll}
\hline $\begin{array}{l}\text { Equilibrium } \\
\text { equations }\end{array}$ & Plasticity conditions & Damage conditions \\
\hline $\begin{array}{lll}\text { Singular } \\
\text { domain } \Gamma\end{array}$ & \\
$\llbracket \sigma \rrbracket=0$ & KKT conditions: & KKT conditions: \\
& $\llbracket\left[\dot{\Lambda} \rrbracket \geq 0, f_{0}^{p} \leq 0,\left[\dot{\Lambda} \rrbracket f_{0}^{p}=0\right.\right.$ & $\dot{d} \geq 0, f^{d} \leq 0, \dot{d} f^{d}=0$ \\
& Yield function: & Yield function: \\
& $f_{0}^{p}:=\left|\sigma_{0}\right|-\sigma_{y 0}$ & $f^{d}:=2(1-d) \sigma_{y 0} \llbracket \Lambda \rrbracket+g_{f} l_{c} \| d^{\prime} \rrbracket$ \\
& Flow rule: & \\
& $\|\dot{u}\|=\operatorname{sign}(\sigma) \llbracket[\dot{\Lambda}]]$ & \\
\hline
\end{tabular}

In addition, Eq. (A4) should be corrected as:

The original article can be found online at https://doi.org/10.1007/ s00466-020-01887-1.

Jianguang Fang

Jianguang.Fang@uts.edu.au; FangJG87@gmail.com

$\triangle$ Chengqing Wu

Chengqing.Wu@uts.edu.au

1 School of Civil and Environmental Engineering, University of Technology Sydney, Sydney, NSW 2007, Australia

2 Institute of Structural Mechanics, Bauhaus-University Weimar, 99423 Weimar, Germany

3 School of Aerospace, Mechanical and Mechatronic Engineering, The University of Sydney, Sydney, NSW 2006, Australia 
$\int_{\Omega}-(\operatorname{div} \boldsymbol{\sigma}+\mathbf{b}) \cdot \delta \mathbf{u d} \Omega+\int_{\partial \Omega}(\mathbf{n} \cdot \boldsymbol{\sigma}-\mathbf{t}) \cdot \delta \mathbf{u d} \partial \Omega=0 \quad$ (A4) $\quad \begin{aligned} & \text { Publisher's Note Springer Nature remains neutral with regard to } \\ & \text { jurisdictional claims in published maps and institutional affiliations. }\end{aligned}$

. 